drei widmen sich einem so aktuellen wie brisanten Themenkomplex mit jeweils unterschiedlicher Schwerpunktsetzung. Während Nachtwey eine historisch-vergleichende Betrachtung parteipolitischer Entscheidungen unter bestimmten institutionellen Bedingungen zum Ausgangspunkt einer Herausarbeitung grundsätzlicher Gerechtigkeitskonzepte macht, liegt der Fokus Walters eher auf rezenten parteipolitischen Entwicklungen. Dass der Wandel sozialstruktureller Faktoren hier eine etwas weniger prominente Berücksichtigung findet, scheint dem geringeren Gesamtumfang der Publikation geschuldet. Lessenich schließlich stellt mit seiner Analyse des flexiblen Sozialstaats aus subjekttheoretischer Sicht eine innovative und scharfsinnige Kritik moderner Subjektivierungsformen unter den sich verändernden wirtschaftlichen Bedingungen vor. Unterschiede ergeben sich hinsichtlich der Berücksichtigung von Globalisierungsentwicklungen auf die Gestaltung sozialdemokratischer Politik. Während die Auseinandersetzung mit diesem Aspekt bei Walter und Lessenich vergleichsweise knapp ausfällt, stellt die Rekonstruktion sozialdemokratischer Sichtweisen auf die Implikationen einer fortschreitenden Internationalisierung der Märkte einen wesentlicheren Teil der Arbeit von Nachtwey dar. Wie bereits angemerkt, zeichnen sich vor allem die Texte von Walter und Lessenich durch einen literarischen Schreibstil aus, der nicht nur die Informationsaufnahme erleichtert: Es macht tatsächlich Spaß, sie zu lesen.

Martin Seeliger

\section{POLITISCHE ÖKONOMIE}

Douglass, C. North, John Joseph Wallis und Barry R Weingast. Violence and Social Orders: A Conceptual Framework for Interpreting Recorded Human History. Cambridge. Cambridge University Press 2009. 308 Seiten.

$19,95 €$.

Douglass North, John Wallis und Barry Weingast haben mit ihrer Monographie „Violence and Social Orders“ einen ambitionierten Beitrag zur institutionentheoretisch fundierten Analyse wirtschaftlicher Entwicklung vorgelegt. Dabei wählen sie eine transdisziplinäre Perspektive, die institutionenökonomische und pluralismustheoretische Argumente miteinander verbindet, und sich damit im analytischen Feld der politischen Ökonomie positioniert. Mit der Fragestellung nach den institutionellen Bestimmungsgründen langfristigen Wirtschaftswachstums knüpfen North, Wallis und Weingast an ein Forschungsprogramm an, das von North bereits in den 1970er Jahren mit der Arbeit „How the West Grew Rich“ umrissen wurde. Dort stand die Rolle von Eigentumsrechten im Mittelpunkt eines paradigmatischen Ansatzes, den North zu einer Transaktionskostentheorie wirtschaftlicher Entwicklung ausbaute. North und Weingast haben diese Überlegungen weitergeführt, indem sie die Lösung des Problems der glaubhaften Selbstverpflichtung als Voraussetzung marktwirtschaftlicher Prosperität identifizierten. Der potentielle Konflikt zwischen staatlichem Gewaltmonopol und der Garantie privater Eigentumsrechte lässt sich über Verfassungsnormen lösen, so dass dem politischen System eine entscheidende 
Rolle für die marktwirtschaftliche Entwicklung zukommt. Bemühte sich North zuletzt um die verhaltenswissenschaftliche Mikrofundierung seiner Entwicklungstheorie, wobei er sich auf kulturell tradierte Wertvorstellungen konzentrierte, so greift er nun in „Violence and Social Orders" zusammen mit seinen langjährigen Forschungspartnern Wallis und Weingast auf die Problematik der glaubhaften Selbstverpflichtung zurück.

In „Violence and Social Orders“ entwickeln North, Wallis und Weingast (im Folgenden: NWW) einen konzeptionelle Rahmen, der die institutionelle Strukturierung kollektiven Handelns im Sinne spezifischer sozialer Ordnungen erfassen soll. Zwei Typen sozialer Ordnungen werden vorgestellt: Ordnungen mit beschränktem Zugang (limited access orders) und Ordnungen mit offenem Zugang (open access orders). Diese Ordnungstypen werden durch historische Differenzen in ihrer ökonomischen Leistungsfähigkeit charakterisiert. Ausgangspunkt ist die These, dass der Zweck von Organisationen in der Aneignung politisch-ökonomischer Renten begründet ist. Soziale Ordnungen mit beschränktem Zugang erlauben nur einen institutionell eingeschränkten Zugang zu den Organisationen eines Gemeinwesens bei gleichzeitiger Ungleichverteilung gesellschaftlicher Gewaltressourcen. Dieser beschränkte Zugang basiert auf sozialen Privilegien, welche die Herausbildung neuer Organisationen insbesondere von Unternehmen - blockieren, was wiederum Elitenrenten stabilisiert. Zwar kann damit das Problem der gewaltsamen Aneignung von Ressourcen eingedämmt werden, zugleich wird aber auch der Marktwettbewerb gehemmt. Soziale Ordnungen mit beschränktem Zugang gehen daher mit relativ geringem Lebensstandard einher. Anders die Ordnungen mit offenem Zugang. Sie verfügen über einen dezentralisierten politischen Steuerungsapparat sowie eine leistungsfähige Verwaltung. Die rechtsstaatlich eingefasste Gleichbehandlung der Gesellschaftsmitglieder unterstützt die Herausbildung neuer Organisationen, vor allem wettbewerbsfähiger Unternehmen. Die Erosion und Neubildung von Organisationsrenten findet in einem Prozess kreativer Zerstörung statt, als schumpeterianischer Entwicklungsprozess. Resultat dieser institutionellen Offenheit ist ein relativ hoher Lebensstandard.

Diese Leistungsprofile resultieren aus institutionellen Unterschieden im Umgang mit der Eindämmung gewaltsamer Ressourcenaneignung. Marktaustausch bedarf der Rechtssicherheit, so dass die Durchsetzung von Eigentumsrechten in arbeitsteiligen Gesellschaften mit der Zuweisung legitimer Gewaltausübung einhergeht. In diesem Zusammenhang wird behauptet, dass die Rentenaneignung durch Organisationen ein zentraler Mechanismus zur Lösung des Gewaltproblems ist. Organisationen können die gewaltsame Rentenaneignung gesellschaftlicher Machteliten durch eine aushandelbare Rentenaneignung ersetzen. Dies eröffnet die Möglichkeit der kulturellen Evolution komplexer Marktgesellschaften mit demokratischen verfassten zivilgesellschaftlichen Strukturen.

Historisch bilden sich zunächst soziale Ordnungen mit beschränktem Zugang heraus, in denen das Gewaltproblem dadurch gelöst wird, dass gesellschaftliche Elitegruppenwechselseitig verpflichtende Vereinbarungen zur Kontrolle ihrer Gewaltressourcen treffen. 
An diesem Punkt greift eine zentrale Vorstellung der institutionalistischen Staatstheorie, die in der historischen Forschung vor allem mit den Arbeiten Tillys verbunden wird: Die produktive Bevölkerung wird von Eliten, die über gewaltförmige Herrschaftsmittel verfügen, vor äußeren Feinden geschützt als Gegenleistung muss sie materiellen Tribut an die Eliten entrichten, etwa in der Form von Steuern. NWW gehen über diese Vorstellung hinaus, indem sie das Kriterium des Zugangs zu gesellschaftlichen Organisationen anfügen. In Ordnung mit beschränktem Zugang bleiben die auf persönlichen Bindungen beruhenden Absprachen zwischen den Elitegruppen fragil. Damit sind den ökonomischen Entwicklungspotentialen enge Grenzen gesetzt. Ordnungen mit offenem Zugang generieren über ihre Innovationsfähigkeit ein langfristig anhaltendes Wirtschaftswachstum. Dies hat mit der für diesen Ordnungstyp spezifischen Form der Kontrolle von Gewaltressourcen durch eigenständige Akteure zu tun, die in Gestalt von Polizei, Militär und Rechtsprechung als formal unparteiische Instanzen auftreten, die an konstitutionelle Regeln gebunden sind. Die Konfliktregulierung ist also nicht mehr personalisiert, sondern über eine umfassende Regelbindung so weit objektiviert, dass die staatlichen Organe eine glaubhafte Selbstverpflichtung zur Regelbefolgung eingehen. Mit dieser Durchsetzung rechtsstaatlicher Prinzipien geht die Öffnung des Zugangs zu gesellschaftlichen Organisationen einher. Dabei ist die rechtlich gesicherte Möglichkeit der Neugründung von Organisationen jenseits exklusiver Privilegien von zentraler Bedeutung. Unternehmensgründungen fördern eine dynamische Wirtschaftsentwicklung.
Politisch orientierte Organisationen beleben zudem den offenen Wettbewerb zwischen Interessengruppen. Auf diese Weise bildet institutionelle Offenheit den Kern funktionsfähiger Demokratien, der typischerweise von außenwirtschaftlicher Offenheit begleitet wird. Damit schließt sich der Kreis zu Hayeks Argumenten über Wissenswettbewerb, Wirtschaftsentwicklung, und kultureller Evolution, auf die North in seinen jüngeren Arbeiten wiederholt verwiesen hat.

Soziale Ordnungen mit offenem $\mathrm{Zu}-$ gang werden schließlich anhand prägnanter Charakteristika umrissen, die einer historisch-empirischen Forschungsskizze dienlich sein sollen. Erstens, gesellschaftlich fest verankerte Wertvorstellungen zur inklusiven Gleichbehandlungen aller Staatsbürger. Zweitens, freier individueller Zugang $\mathrm{zu}$ wirtschaftlichen, politischen, religiösen und bildungsbezogenen Aktivitäten. Drittens, Organisationsfreiheit in all diesen Aktivitäten. Viertens, rechtsstaatliche Gleichbehandlung vor dem Gesetz als Garantie bürgerlicher Freiheiten. Fünftens, unpersönlich-versachlichte Austauschbeziehungen. Aus diesen Charakteristika erschließen sich unmittelbar die Ordnungsfunktionen des kombinierten politischen und ökonomischen Wettbewerbs. Den unpersönlichen Austauschbeziehungen auf offenen Märkten entspricht die versachlichte politische Kontrolle der Gewaltressourcen mit ihrer Trennung von politisch-administrativer und polizeilich-militärischer Exekutive. Der Staat erscheint als „Organisation von Organisationen ". Mit dieser pluralistischen Sichtweise wenden sich NWW explizit gegen die Modellierung des Staates als uniformem Einzelakteur. Tatsächlich ist die Betonung pluralistischer Theo- 
riesegmente in der ganzen Arbeit präsent, insbesondere im Hinblick auf Dahls Thesen zur politischen Machtbalance zwischen Interessengruppen. Damit wenden sich NWW gegen Mancur Olsons institutionenökonomische Kritik der Pluralismustheorie. So betonen NWW die Notwendigkeit eines endogenen Ansatzes, der die strategische Selbstbeschränkung von Interessengruppen auf offenen politischen Märkten betont. Das Kalkül der Marktbestreitbarkeit verhindert die von Olson behauptete Tendenz zur institutionellen Sklerose des Marktsystems durch anhaltendes Rent-Seeking.

Angesichts der von NWW getroffenen Einschätzung, dass aktuell nur 15 Prozent der Weltbevölkerung in zusammen 25 Ländern in offenen Ordnungen leben, während die überwältigende Mehrheit sozialen Ordnungen mit beschränktem Zugang angehört, stellt sich die Frage nach den institutionellen Voraussetzungen des Übergangs zu Ordnungen mit offenem Zugang. Drei Bedingungen müssen hierfür erfüllt sein. Erstens, gesellschaftliche Eliten müssen sich einer verpflichtenden Regelbindung unterwerfen, die sukzessive auch auf die Gesamtbevölkerung auszudehnen ist. Zweitens, es kommt zur Herausbildung von Elitenorganisationen, die eine eigene Rechtspersönlichkeit entwickeln und damit unabhängig von konkreten Personen existieren können. Drittens, die Eliten üben eine kollektive politische Kontrolle über das Militär aus und lösen damit das gesellschaftliche Gewaltproblem. Diese thesenhaft skizzierten Übergangsprozesse werden von NWW anhand historischer Fallstudien illustriert. So dient das karolingische Reich als Beispiel früher Staatenbildung, während die Entwicklung des englischen Boden- rechts zur Erläuterung der Sicherung von Eigentumsrechten angeführt wird. Im Hinblick auf die Dynamik offener Ordnungen werden historische Pfade in der Entwicklung des Gesellschaftsrechts in Großbritannien, Frankreich, und den Vereinigten Staaten angeführt. Das Fazit des Buches bezieht diese historische Evidenz auf aktuelle Problemlagen angewandter politischer Ökonomie: der Einfluss politischer Demokratie auf die wirtschaftliche Entwicklung darf nicht auf den Faktor freier Wahlen reduziert werden, vielmehr muss auch die organisationale Offenheit einbezogen werden.

Eine kritische Würdigung des von NWW vorgelegten Ansatzes muss zunächst anerkennen, dass es den Autoren gelingt, institutionenökonomischen Thesen mit pluralismustheoretischen Argumenten anzureichern, und damit das vor allem mit Douglass North verbundene Forschungsprogramm zur institutionellen Dynamik wirtschaftlicher Entwicklung weiterzuentwickeln. Ob es allerdings gelingt, mit diesem Unterfangen eine „neue Forschungsagenda für die Sozialwissenschaften" vorzulegen, wie von den Autoren plakativ behauptet, muss allerdings bezweifelt werden. In kritischer Hinsicht ist zunächst zu vermerken, dass NWW keinen präzisen theoretischen Kern für ihr Modell sozialer Ordnungen ausarbeiten. Die wiederholt angeführten verhaltenswissenschaftlichen und spieltheoretischen Bezüge können das Fehlen einer stringenten Handlungstheorie nicht aufwiegen, so dass die Mikroebene des Ansatzes unbestimmt bleibt. Dieses Defizit wird anhand der Frage der Organisation von Interessengruppen besonders deutlich. Das in Olsons Logik des kollektiven Handelns analysierte Phänomen der asymmetrischen 
Organisationsfähigkeit gesellschaftlicher Interessengruppen lässt sich keinesfalls allein durch die Berücksichtigung des offenen Marktzugangs aufheben. NWW eröffnen zwar mit ihrer Forderung nach einer Endogenisierung von Olsons Interessengruppenmodell eine fruchtbare Diskussion, aber dennoch bleibt Olsons Kritik des Pluralismus relevant. Zudem bleibt der politische Wettbewerb in der Darstellung von NWW eher unterbelichtet. Politische Parteien werden als spezifische Organisationen kaum wahrgenommen, so dass auch die politische Wettbewerbsdynamik unberücksichtigt bleibt. Schließlich mangelt es dem von NWW vorgelegten Entwurf an empirischer Evidenz. Die historischen Fallstudien können zwar die vorgelegten konzeptionellen Zusammenhänge beleuchten, vertiefendes statistisches Material und entsprechende quantitative Analyseoptionen werden jedoch explizit ignoriert. In diesem Sinne ist „Violence and Social Orders" ein wichtiger Beitrag zur institutionalistischen Staatstheorie - fällt dabei aber eher durch weiterführende Fragen, als durch empirisch fundierte Antworten auf.

Alexander Ebner

\section{HISTORISCHE POLITIKFORSCHUNG}

\section{Bleek, Wilhelm. Friedrich Christoph \\ Dahlmann. Eine Biographie. München. C. H. Beck 2010. 472 Seiten. 34,95€.}

Friedrich Christoph Dahlmann gehört zu den großen Gestalten des deutschen Konstitutionalismus im 19. Jahrhundert: Geboren 1785 in Wismar, Altphi- lologe, Lehrer der „Politik“, wie sein Buch von 1835 heißt, in dem die Politik „auf den Grund und das Maaß der gegebenen Zustände zurückgeführt" wird, Teil der nationalen Bewegung des frühen 19. Jahrhunderts, Verfasser des Hannoverschen Staatsgrundgesetzes von 1833, eine, nein: die entscheidende Figur der „Göttinger Sieben“, die gegen die Aufhebung eben dieses Grundgesetzes protestierten, Berufung an die Bonner Universität und Mitglied der Frankfurter Nationalversammlung. Dahlmann ist Gelehrter und Reformer, ein „politischer Professor“, der der Verfassungs- und Nationalbewegung Gestalt und Gehalt gab, der mit ihr aber auch scheiterte.

Dahlmann ist heute so gut wie vergessen. Nur Verfassungshistorikern und Politikwissenschaftlern, die sich der Geschichte des eigenen Faches annehmen oder versichern wollen, ist Dahlmann ein Begriff. Dahlmann studierte die alten Sprachen, kannte sich im antiken Griechenland hervorragend aus, suchte aus der Geschichte Maßstäbe für seine Gegenwart zu gewinnen. Er war ein Wissenschaftler des Politischen, dem es um die "gute Ordnung“ des Gemeinwesens geht. Die Wissenschaft sollte gleichermaßen realistisch wie praktisch sein, ihren empirischen Bezug in den „gegebenen Zuständen“ finden, den "Grund“ der jeweiligen Ordnung historisch erschließen und für eine Ordnung der „Mitte und des Maßes “ plädieren. Dahlmann war ein Ethiker, der feste Überzeugungen besaß und im Gewissen den Maßstab seiner Urteilskraft fand. Seine politischen Positionen schärften sich, als er 1809 mit Heinrich von Kleist von Dresden in Richtung Wien aufbrach und auf dem Schlachtfeld von Aspern die Leichenberge inspizierte. In Dresden hatte er 